\title{
A Novel Dressing Method Using a Silicone Sheet to Maintain Distant Abdominal Flaps after Reconstruction of Finger Skin Defects
}

\author{
Jae Kyoung Kang, Jae Seong Lee, Myoung Soo Shin, Byung-Min Yun \\ Department of Plastic and Reconstructive Surgery, Jeju National University College of Medicine, Jeju, Korea
}

\begin{abstract}
The distant abdominal flap has long been used in hand reconstruction due to its stability and minimal complications at the donor site. After the distant abdominal flap is performed, the hand should remain attached to the abdomen for 3 weeks. Keeping this attachment stable without any kinking of the flap is crucial. In this report, a distant abdominal flap was used to reconstruct defects in skin on a finger after trauma, and a silicone sheet was used to reinforce the area as to prevent kinking of the abdominal flap. The flap was successfully kept in position without kinking or tension until it was detached. Reinforcing a flap with a silicone sheet may work as an effective wound dressing method that prevents kinking in pedicle flaps.
\end{abstract}

Keywords: Surgical flaps; Silicone; Wound healing

\section{Introduction}

While a distal abdominal flap is highly stable, arterial necrosis of the flap margin has been reported as a complication [1]. Until the flap is detached, it should remain attached to the donor and recipient sites in position for 3 weeks, during which the flap base is prone to kinking or torsion. Since flap kinking and torsion may result in flap necrosis, it is important to maintain the position of the flap postoperatively [2]. We report two cases in which we used a distant abdominal flap to reconstruct defects in finger skin, reinforced the pedicle by using a silicone sheet which is commonly used for nose and ear dressing [3], and were able to easily keep the flap stable after surgery. We also sought to determine whether this dressing method would be useful in maintaining the position of not only a distant abdominal flap but also a pedicle flap, where the pedicle is susceptible to kinking or torsion after reconstruction. The study was performed in accordance with the principles of the Declaration of Helsinki. Written informed consents were obtained.

\section{Ideas}

\section{Idea 1}

A 55-year-old man with no notable medical history visited the hospital after his left hand had been sucked into a machine, resulting in skin defects in his left thumb and index finger. While his left thumb had a distal phalanx tip defect with the bone exposed, there was no fracture. His left index finger had a $3.5 \times 1.5 \mathrm{~cm}$ volar aspect skin defect across the distal phalanx and the middle phalanx, and the tendon was exposed,

\section{Idea and Innovation}

Received: June 30, 2019

Revised: July 29, 2019

Accepted: July 30, 2019

\section{Corresponding author:}

Byung-Min Yun, M.D., Ph.D.

Department of Plastic and Reconstructive Surgery, Jeju National University College of Medicine, 102 Jejudaehak-ro, Jeju 63243, Korea

Tel: +82-64-717-1742

Fax: +82-64-755-6130

E-mail: almostfree@hanmail.net

This is an Open Access article distributed under the terms of the Creative Commons Attribution Non-Commercial License (http://creativecommons.org/licenses/by-nc/4.0/) which permits unrestricted non-commercial use, distribution, and reproduction in any medium, provided the original work is properly cited.

C) 2019 Korean Wound Management Society 
but the tendon and bone were still intact. With the patient under general anesthesia, we performed a full-thickness skin graft on his index finger, and elevated a $3 \times 10 \mathrm{~cm}$ abdominal flap to cover his thumb skin defect. We sutured the flap pedicle into a tubular shape. Because the flap base can easily kink at an acute angle when reconstructing a defect in the dorsal aspect (Fig. 1), we designed a reinforced silicone sheet (Bioplexus, Ventura, CA, USA) that would hold the flap in position to prevent kinking. The sheet was designed to be twice as wide as the tubular pedicle and to be of the same length as the total flap. Half of the sheet was placed on the elevated flap, and the other half was placed on non-elevated abdominal skin (Fig. 2). This flexible sheet of silicone was attached to the abdomen skin and the flap by skin stapler (Fig. 3). We applied wet dressings to the

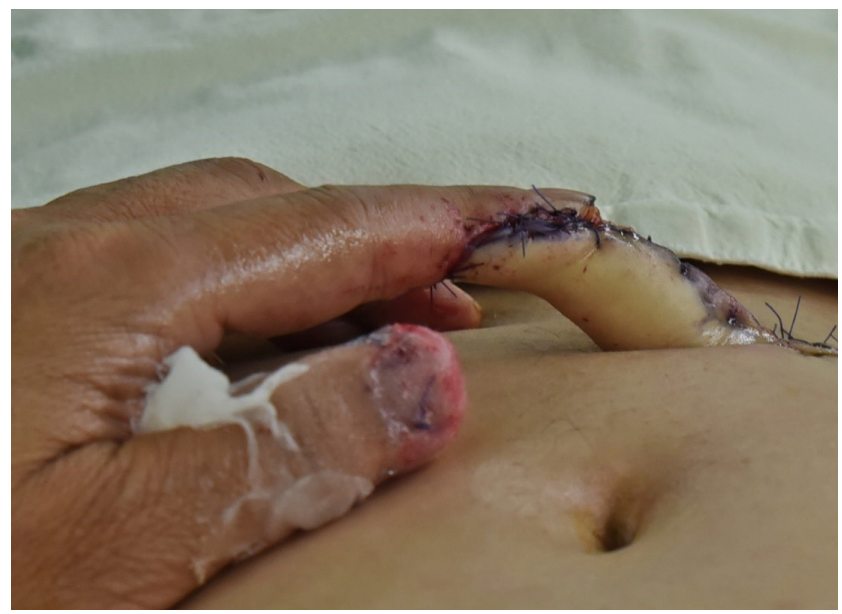

Fig. 1. Immediate postoperative view. The flap base is kinked at an acute angle.

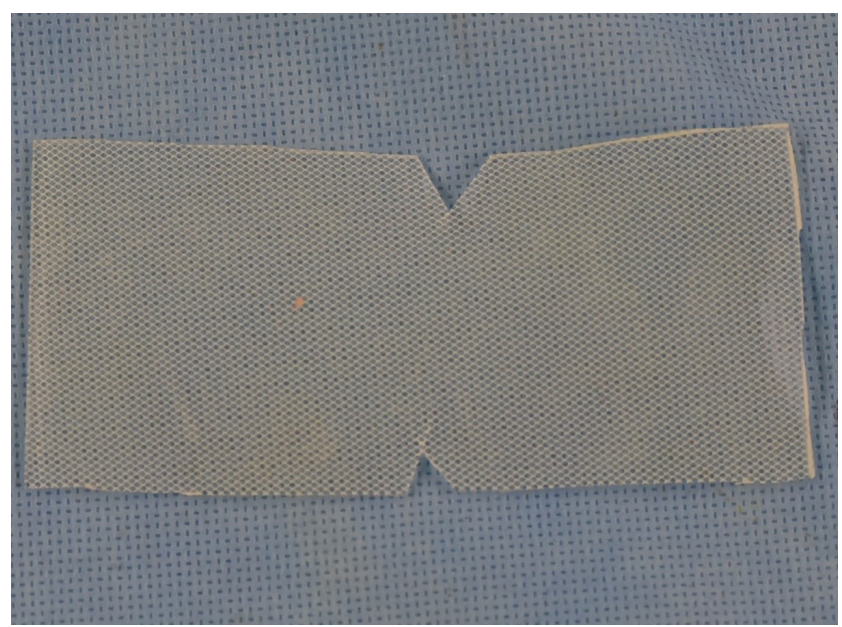

Fig. 2. The dressing material of idea 1 . The silicone sheet (Bioplexus, Ventura, CA, USA) is designed to fit the size of the flap. exposed subcutaneous tissue on the other side, using Vaseline gauze. We also put pads under his axilla, forearm, and palm, held them in position with tape, and maintained the angle of his hand. Three days after surgery, we removed the pads, kept the silicone sheet in place, and immobilized his arm using only elastic bandages. We provided daily Vaseline wet dressings for the first week and betadine ointment dressings every other day for the following 2 weeks. The silicone sheet was maintained until flap detachment on postoperative day (POD) 21. For 3 weeks, the flap was well maintained without any complication.

\section{Idea 2}

A 50-year-old man with no notable medical history visited the hospital for distal phalanx amputation of his right middle fin-

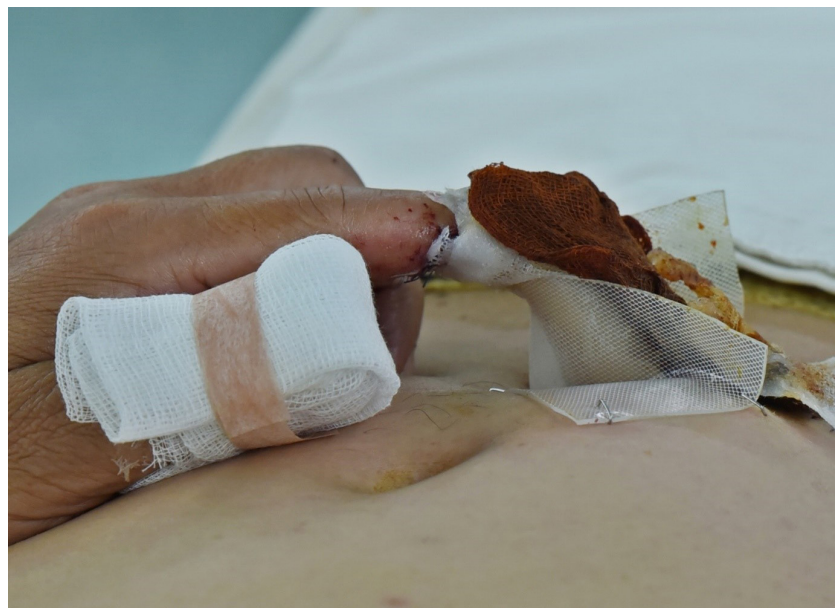

Fig. 3. The dressing method of idea 1 . The reinforced silicone sheet is immobilized under the flap to prevent kinking.

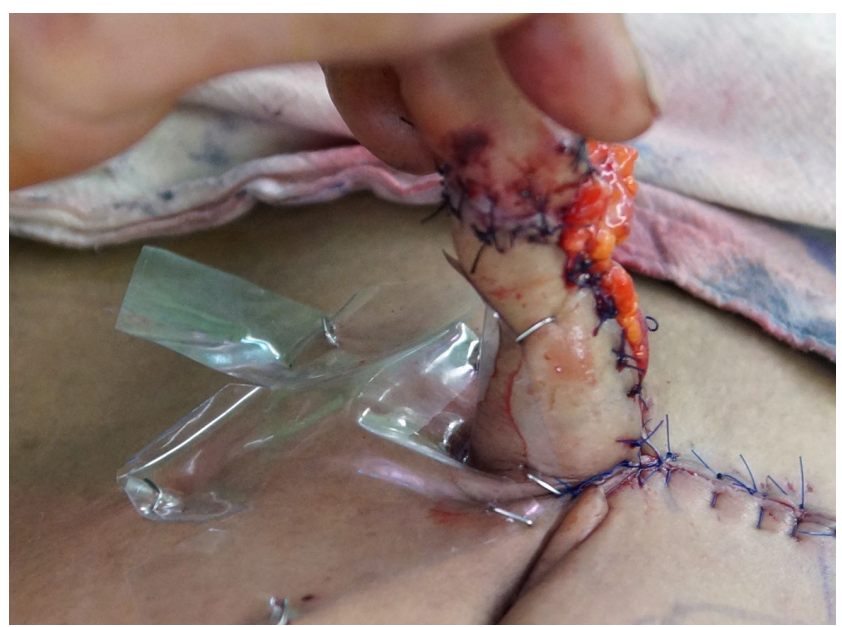

Fig. 4. The dressing method of idea 2. The non-reinforced silicone sheet is immobilized under the flap to prevent kinking. 
ger and skin defects on the volar aspect across the distal phalanx of his right ring finger, after his fingers had been caught underneath a tractor falling sideways. With the patient under general anesthesia, we elevated a $6 \times 10 \mathrm{~cm}$ abdominal flap and covered skin defects on his middle and ring fingers with the abdominal flap. Then we designed a non-reinforced silicone sheet as wide as the tubular width and as long as the length of the flap and immobilized it by with staples (Fig. 4). We provided daily Vaseline wet dressings for the first week and betadine ointment dressings every other day for the next 2 weeks. The silicone sheet was maintained until flap detachment on POD 22. For 3 weeks, the flap was well maintained without any complication.

\section{Discussion}

Complications that may occur from reconstruction using a distant flap include marginal necrosis, wound dehiscence, hematoma, and infection [4]. Flap necrosis may be caused by a flap much longer than the pedicle, the flap detaching too early, unfavorable blood vessel conditions of the recipient site, or hematoma clotted under the flap. In a random flap, which receives blood from the subdermal plexus just as a distant flap would, kinking in the flap base or tension created by the stretched flap could cause necrosis [3]. Such pedicle flap necrosis is more often caused by problems with the venous drainage system than because of arterial insufficiency [3,5]. This leads to flap edema, a purple-blue colored flap, or dark colored bleeding after a pin prick test [6]. In particular, the

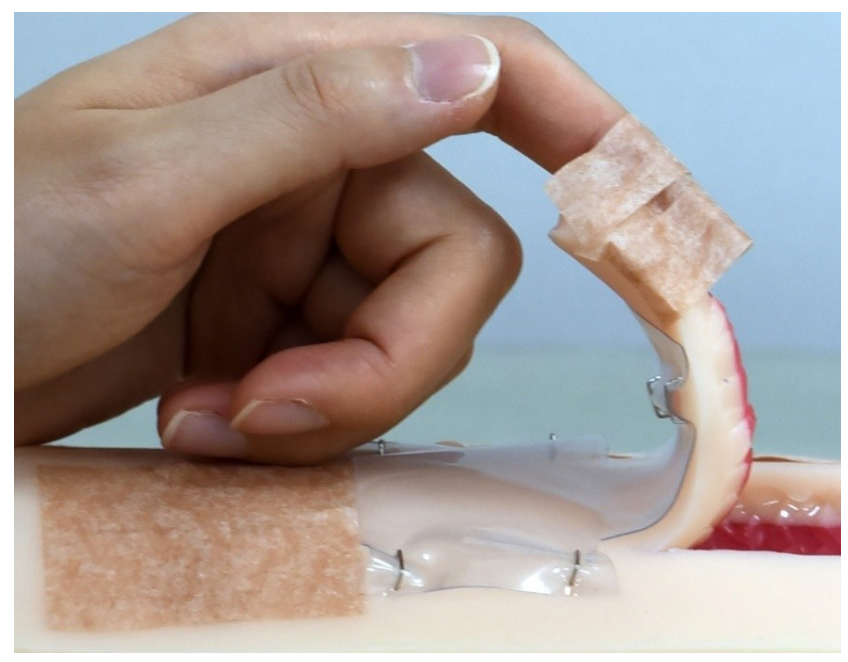

Fig. 5. A simulation of the dressing method. pedicle can kink easily at an acute angle when a volar aspect defect is covered with the distant flap, as in the present cases. Therefore, it should be covered with caution.

Previous studies suggested flap tubing to prevent pedicle kinking and to reduce the raw area. The rigidity of the flap tubing prevents kinking of the flap, and a reduced raw area can decrease the risk of infection [5,7]. However, the drawback is that tubing makes flap contouring difficult and interferes with the flow of blood [7].

Maintaining a stable posture for 3 to 4 weeks is also important in preventing the pedicle from kinking too much. Previous studies used adhesive bandages or plaster tape to immobilize the arm by placing a pillow under the axilla and hand $[2,8]$. In addition, three lines were drawn from the wrist to the belly, and the patients and their caregivers were instructed to keep the posture steady with the lines matched [8]. In the present cases, cotton pads were also placed under the axilla, forearm, and hand until POD 7 to hold the arm in position, using elastic bandages and plaster. From POD 7 until detachment, elastic bandages were used to immobilize the upper arm and trunk, and an arm sling was applied.

In the present study, both patients had skin defects on the volar aspect, meaning the flap pedicle could kink at an acute angle. To address the kinking, we reinforced it with a flexible silicone sheet (which could also be supplemented for rigidity) at the site where the flap pedicle was kinked. The figure is a simulation of our dressing method (Fig. 5). After POD 7, plaster was replaced with elastic bandages to help to keep the flap pedicle stable, while also allowing slight movement of the arm.

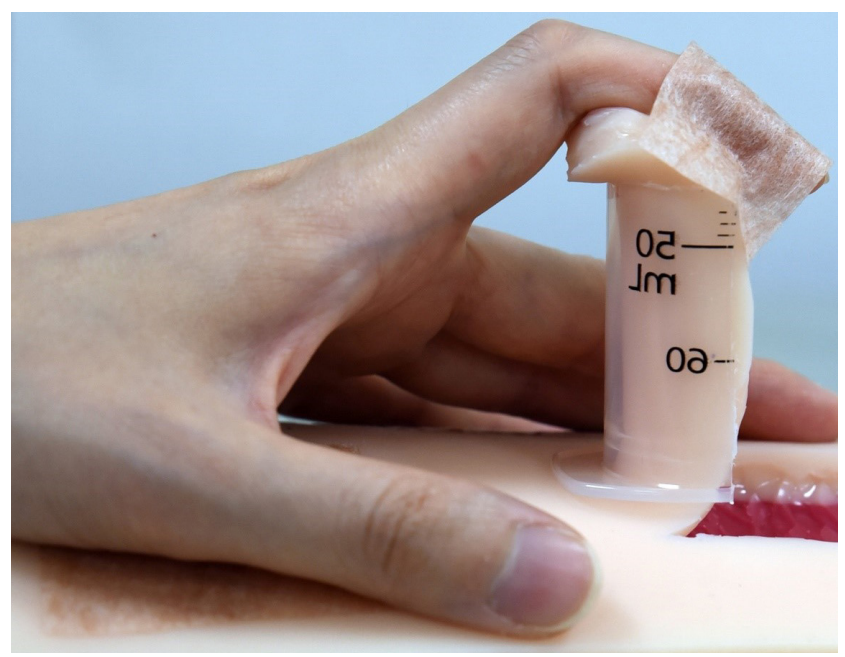

Fig. 6. An alternative dressing method. A 50- $\mathrm{mL}$ syringe could be used as dressing material. 
Another alternative for reinforcing the flap could be to split in half a $50-\mathrm{mL}$ syringe, which is easily available in the operating room (Fig. 6). Unlike a silicone sheet, the syringe is rigid and helps maintain the flap in place by propping up the flap base. This is especially helpful for pediatric patients who have severe irritability after surgery. But this reinforcement can also put pressure on the flap, and the flap could be detached if the material is too rigid or the shape of material does not fit the flap. Therefore, appropriate material should be chosen, and it should also be designed so that it does not put undue pressure on the flap.

We believe that the aforementioned dressing method can be applied not only with a silicone sheet but also other easily available materials. In addition, by preventing kinking and acute angle flexion in not only a distant abdominal flap but also other types of delayed pedicle flaps, including cross leg flaps, cross finger flaps, forehead flaps, and thenar flaps, this dressing method is also expected to prevent local complications.

\section{Conflict of interest}

No potential conflicts of interest relevant to this article are reported.

\section{Acknowledgments}

Jae Kyoung Kang https://orcid.org/0000-0003-3547-6845
Jae Seong Lee https://orcid.org/0000-0002-0087-8791

Myoung Soo Shin https://orcid.org/0000-0002-8450-821X

Byung-Min Yun https://orcid.org/0000-0002-3545-4446

\section{References}

1. Sabapathy SR, Bajantri B. Indications, selection, and use of distant pedicled flap for upper limb reconstruction. Hand Clin 2014;30:185-99.

2. Dawson RL. Compound fractures of the lower limb with skin loss: complications of the cross-leg flap operation. J R Soc Med 1972;65:626.

3. Godley FA. Experience with the malleable ear dressing, a versatile silicone-lined bandage for the auricle. Ear Nose Throat J 2003;82:570-4.

4. McGregor IA, Jackson IT. The groin flap. Br J Plast Surg 1972;25:3-16.

5. Derganc M, Zdravic F. Venous congestion of flaps treated by application of leeches. Br J Plast Surg 1960;13:187-92.

6. Utley DS, Koch RJ, Goode RL. The failing flap in facial plastic and reconstructive surgery: role of the medicinal leech. Laryngoscope 1998;108(8 Pt 1):1129-35.

7. Bajantri B, Latheef L, Sabapathy SR. Tips to orient pedicled groin flap for hand defects. Tech Hand Up Extrem Surg 2013;17:68-71.

8. Arner M, Moller K. Morbidity of the pedicled groin flap: a retrospective study of 44 cases. Scand J Plast Reconstr Surg Hand Surg 1994;28:143-6. 